Egyptian

Orthodontic Journal

\title{
BISPHOSPHONATE EFFECTS UPON RELAPSE OF ORTHODONTICALLY MOVED TEETH
}

\author{
Hisham I. Osman* and Ahmed Rami Afify
}

ABSTRACT

This study was designed to identify the effects of bisphosphonate (BP) administration on structure and functions of osteoclasts in alveolar bone resorption during relapse after orthodontic movement of rat molars. Twenty four male rats were used in this study. To produce orthodontic force, elastic band was inserted between the upper first and second molars for 21 days. At one day before elastic band removal, bisphosphonate was administered intraperitoneally. After elastic band removal, the rats were further maintained for 0,5 and 10 days. The distal relapse movement of the first molars was examined by means of light, scanning and transmission electron microscopy. In bisphosphonate-administered rats, there was a decrease in the distal relapse movement in comparison to the control group and the histological observations revealed reduction in osteoclasts aggregation along the alveolar bone surfaces opposite the pressure side of the periodontal ligament with degenerative structural changes especially at the fifth day. It was concluded that systemic administration of bisphosphonate decreases the initial relapse in experimentally moved rat molars through a mechanism involving impairment of the structure and resorptive functions of osteoclasts.

\section{INTRODUCTION}

In orthodontic treatment, undesirable movement of anchor teeth during tooth movement and relapse of moved teeth to their original positions after treatment are the major causes of unsuccessful results. Alveolar bone is a unique compartment of

\footnotetext{
* Assistant professor of oral biology, faculty of dentistry, Alexandria University

** Assistant professor of orthodontics, faculty of dentistry, Mansoura University
} 
the skeleton. Its existence depends on the presence of teeth and the maintenance of masticatory function. Uniquely, both the soft and mineralized paradental tissues are highly responsive to external mechanical stresses. This characteristic forms the biological basis of orthodontic and dentofacial orthopedic treatment, as well as guided tissue regeneration. ${ }^{(1)}$

The relapse of moved teeth is a physiologic response of the supporting tissues to force application and is considered to be attributable mainly to the occlusal stability and the increased mechanical tension exerted by the transseptal fiber system which though to be important for stabilizing tooth position and is the apparent source of the stress that leads to relapse of moved teeth. ${ }^{(2,3)}$ Therefore appropriate anchorage preparation and proper retention are essential. However, these problems are often unavoidable because of limitations of the force system; denture conditions, and / or lack of proper cooperation from patient.

To prevent such relapse clinically, long-term mechanical maintenance of moved teeth in the correct position by means of retainers has been widely applied. ${ }^{(4)}$ If undesirable tooth movement could be prevented, such as through chemical blockers of bone resorption, the orthodontic force system would be simpler and more secure However, few studies have been performed to test such blockers to decelerate or inhibit tooth movement. ${ }^{(5)}$

Recently, various bisphosphonates have been developed and successfully introduced to treat patients with metabolic bone diseases, such as osteoporosis, Paget's disease of bone and hypercalcemia with malignancy, which are all attended by increased bone resorption. Bisphosphonates, a class of recently developed drugs, are chemically analogues of pyrophosphate, and are known to inhibit osteoclastic bone resorption, either by direct suppression of osteoclasts, or indirectly by inhibition of secretion of soluble osteoclast activating factors by osteoclasts. ${ }^{(6,7)}$

Bisphosphonates have a P-C-P bond instead of P-O-P bond of inorganic pyrophosphate, which makes them resistant to enzymatic degradation, and gives them a high affinity for hydroxyapatite crystals and inhibit their dissolution, ${ }^{(8)}$ it has also been stated that they inhibit lactic acid production, and the activity of lysozomal enzymes. These factors alone or in combination may contribute to the alternations seen in osteoclast function ${ }^{(9)}$.

However, the precise effects of $\mathrm{BP}$ on the structure and functions of osteoclasts and other bone cells remain unclear. ${ }^{(10)}$

The aim of the present work was to examine the effects of bisphosphonate (BP), pamidronate administration on structure and functions of osteoclasts in 
alveolar bone resorption during relapse of molar teeth in rat, after application of orthodontic force.

\section{Material and methods}

Twenty four male rats (7-weeks-old) were used in this study. At the beginning of the work, a piece of orthodontic elastic band was inserted between the first and second molars in both the right and left maxillae according to the method of Waldo $(11,12)$ and left in place for 21 days. Once the elastic band had been removed, the rats were further maintained for 0,5 and 10 days. One day before elastic band removal, bisphosphonate dissolved in physiologic saline was administered intraperitoneally to the animals of the experimental groups $(0.01 \mathrm{mg} / \mathrm{kg})$. The same volume of physiological saline solution was injected into the control group.

The bisphosphonate $(\mathrm{BP})^{*}$, pamidronate disodium used in this study is disodium 3-amino-1- hdroxypropylidene 1, 1-bisphosphonate pentahydrate.

The rats were classified into 6 groups (each containing 4 rats) according to the day of scarification from the time of bisphosphonate injection:

1. Control group at day 0

2. Control group at day 5

3. Control group at day 10

4. BP group at day 0

5. BP group at day 5

6. BP group at day 10

The rats were anesthetized and killed. Their maxillae were dissected out, cut into two halves and fixed in $2.5 \%$ glutaraldehyde in $0.1 \mathrm{M}$ sodium cacodylate buffer ( $\mathrm{pH}$ 7.3). One half of dissected maxillae were prepared for scanning electron microscopy examination and the scanning micrographs were used for measuring the interval between the first and second maxillary molars. Data were saved on an Excel spreadsheet and then transferred to SPSS software package for statistical analysis. The mean and standard deviation of both groups were calculated. Independent t- test was performed to verify the differences between groups. While the other half of maxillae were prepared for light microscopic examination using $\mathrm{H} \& \mathrm{E}$ stain and transmission electron microscope examination.

\footnotetext{
* Aredia; Ciba-Geigy, Switzerland.
} 
Egyptian

Orthodontic Journal

\section{RESULTS}

\section{Scanning electron microscopic findings: (table 1)}

The distal relapse of the mesially moved first molars following removal of the elastic band after 21 day of insertion were observed from the occlusal surface and resulted in a decrease in the interdental distances between the first and second molars, therefore the shortest interdental distance between the proximal surfaces of the first and second molars in the control groups and bisphosphonate (BP) groups were measured and tabulated.

In the control groups (saline administration), at day 0 , the interdental distance between the first and second molars was $419 \mathrm{um}$. This interdental distance decreased rapidly with relapse reaching 99 um by 5 days, and then diminished at a slower rate, reaching 53 um by day 10

In bisphosphonate (BP) treated rats, the mean of interdental distance on day 0 was 428 um, but was 290 um by 5 days and 106 um by day 10 .

Table 1.

\begin{tabular}{|c|c|c|c|c|c|}
\hline \multirow{2}{*}{} & \multicolumn{2}{|c|}{ Control group } & \multicolumn{2}{c|}{ BP group } & \multirow{2}{*}{ P-value } \\
\cline { 2 - 5 } & $\mathbf{X}$ & SD & $\mathbf{X}$ & SD & \\
\hline Day 0 & $419 \mathrm{um}$ & \pm 38.5 & $428 \mathrm{um}$ & \pm 18.65 & 0.689 \\
\hline Day 5 & $99 \mathrm{um}$ & \pm 10.52 & $290 \mathrm{um}$ & \pm 13.34 & $0.000^{* * *}$ \\
\hline Day 10 & $53 \mathrm{um}$ & \pm 6.7 & $106 \mathrm{um}$ & \pm 6.27 & $0.000^{* * *}$ \\
\hline
\end{tabular}

\section{Light microscopic findings:}

Due to distal relapse of the first molars after removal the elastic band, the PDL on the distal sides of the mesial roots was considered to be the pressure side and the mesial side of the mesial roots to be the tension side.

In control rats (fig.1, 2), on the pressure sides at day 0 and 5 there were numerous deeply located osteoclasts occupying Howship's lacunae along the irregular alveolar bone surface, while on the tension sides (fig. 3), dense and regular arranged collagen fibers were distributed with absence of bone resorption. The alveolar bone surface is regular and covered by cuboidal cells.

In bisphosphonate-treated rats (fig 4,5), fewer osteoclasts were observed along the alveolar bone surface in comparison with the control group on the pressure side 
At day 5, the distribution of osteoclasts in BP treated rats was different from that in untreated controls. Specifically, most of the osteoclasts were clearly localized around blood vessels in vascular canals of alveolar bones. At day 10, a similar histological appearance was observed at the pressure side exhibiting fewer superficially located osteoclasts with increase vascularity (fig. 6). On the tension side, the principle fiber bundles were regularly arranged and the bone surface was relatively smooth with absence of osteoclastic lacunae.

\section{Transmission electron microscopic findings:}

In the control group, areas of active osteoclastic resorption with multinucleated osteoclasts in their lacunae were found in the pressure sites after 5 days of elastic band removal. The osteoclasts exhibit well-developed ruffled borders and clear zones facing the alveolar bone surface. Mitochondria were distributed throughout the cytoplasm together with the presence of numerous vesicles and vacuoles. (fig.7)

In bisphosphonate- treated rats groups at the pressure side, the osteoclasts showed variety of irregular cell configurations on the alveolar bone surface (fig.8, 9, $10 \& 11)$. They have well-developed ruffled borders and clear zones as in control groups. Some osteoclasts displayed clear zones and irregular formed ruffled border with various sizes and configurations. Other osteoclasts showed only clear zones with reduced ruffled borders. Degenerated osteoclasts filled with vacuolar cytoplasm and pyknotic nuclei were also observed. While others were located on bone matrix showed complete absence of ruffled borders and clear zones.

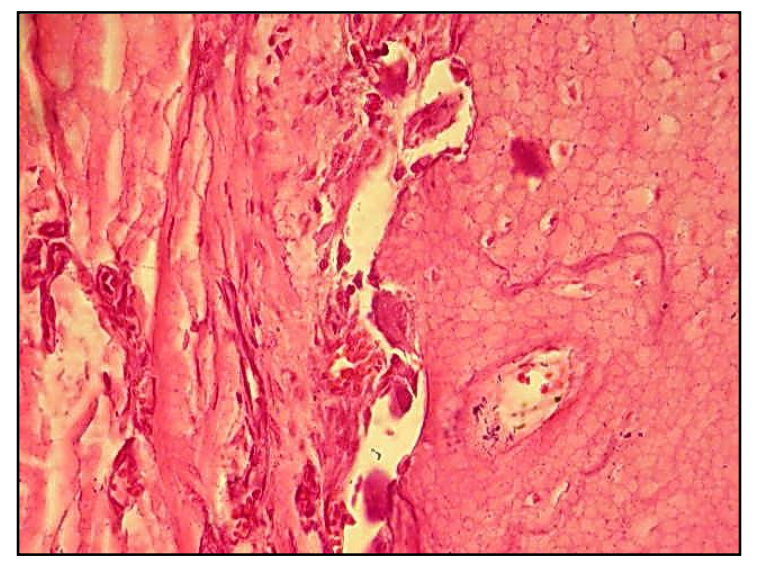

Fig. 1 Control group at day 0, pressure side of the PDL showing the presence of numerous resorbing lacunae along the alveolar bone surface and disorganized fiber bundles $\mathrm{H} \& \mathrm{E} \mathrm{X} 400$ 


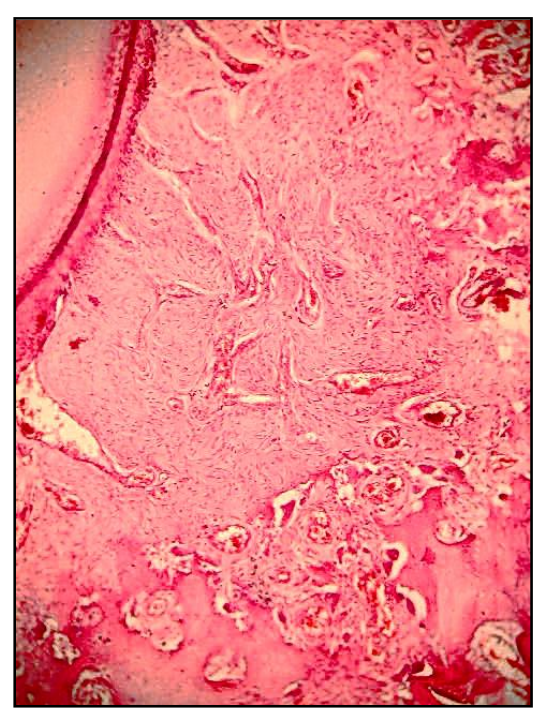

Fig.2 Control group at day 5, pressure side of the PDL. Note the presence rough irregular bone surface covered by multiple Howship's lacunae containing osteoclast cells causing bone resorption $\mathrm{H} \& \mathrm{E}$ X 100

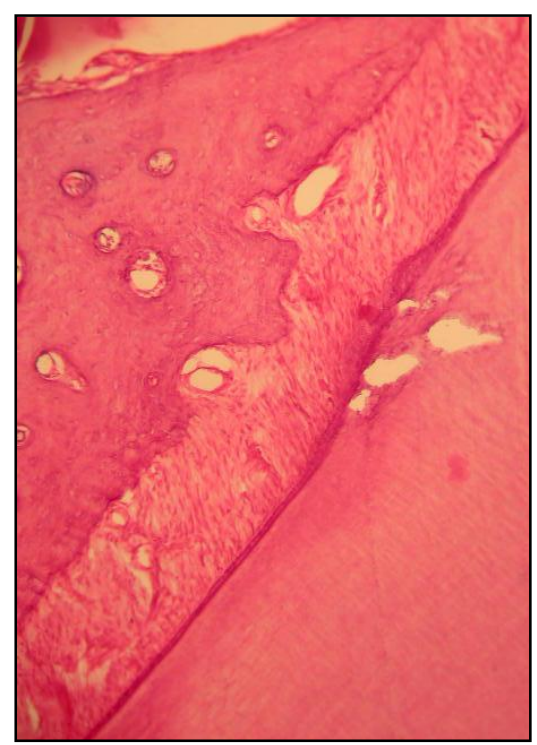

Fig. 3 Control group at day 5 of elastic band removal, tension side of the PDL showing regular distribution of the principle fibers of the PDL and numerous periodontal ligament cells. The alveolar bone surface is relatively regular and coated with osteoblast cells $\quad$ H\&E X 100 
Egyptian

Orthodontic Journal

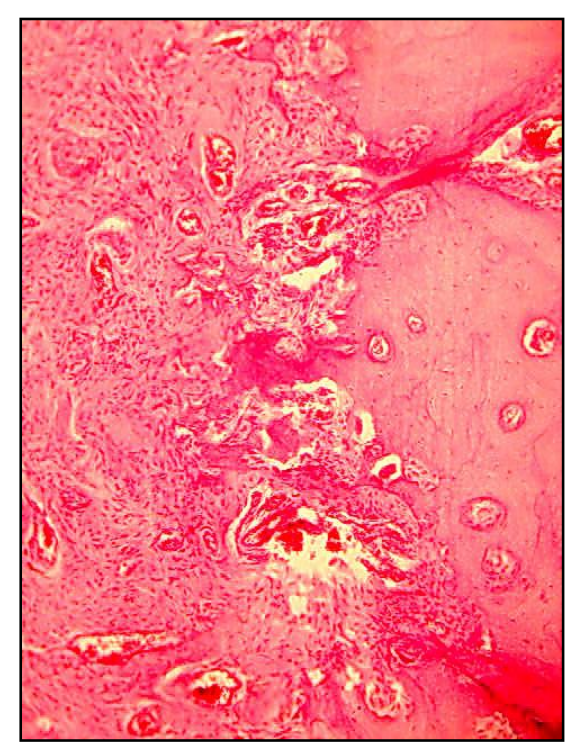

Fig.4 Bisphosphonate- treated rats at day 0 in the pressure side of the PDL showing accumulation of numerous osteoclastic lacunae inducing bone resorption H\&E X 400

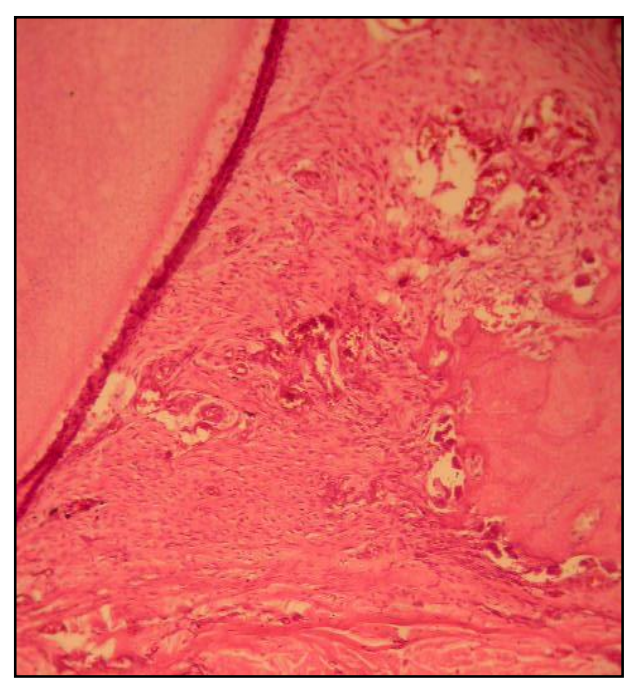

Fig.5 Distal surface of the mesial root of the first molar (pressure side) of bisphosphonatetreated rats at day 5 after elastic band removal showing bone resorption. Osteoclast cells are seen along the alveolar bone surface with little bone resorption. The PDL exhibits increase vascularity $\mathrm{H} \& \mathrm{E}$ X 100 . 


\section{Egyptian}

Orthodontic Journal

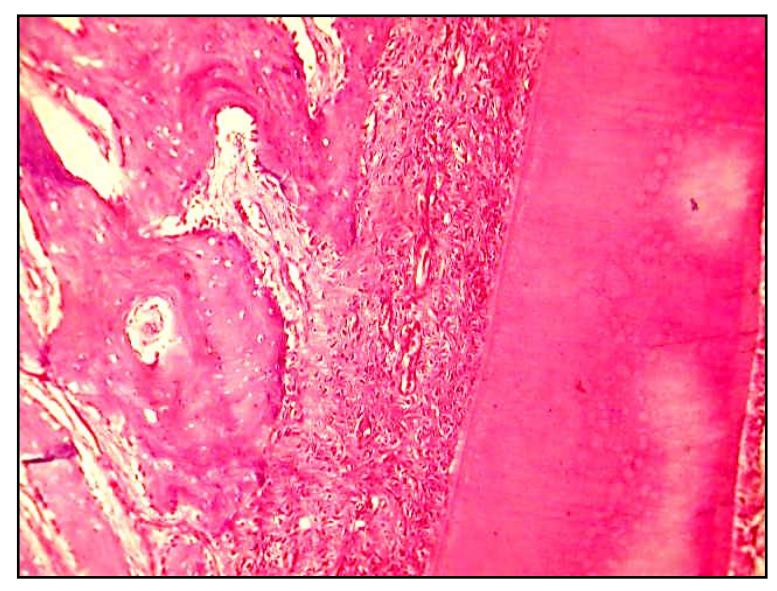

Fig.6 Bisphosphonate-treated rats at day 10 in the pressure side showing relatively absence of bone resorption X100

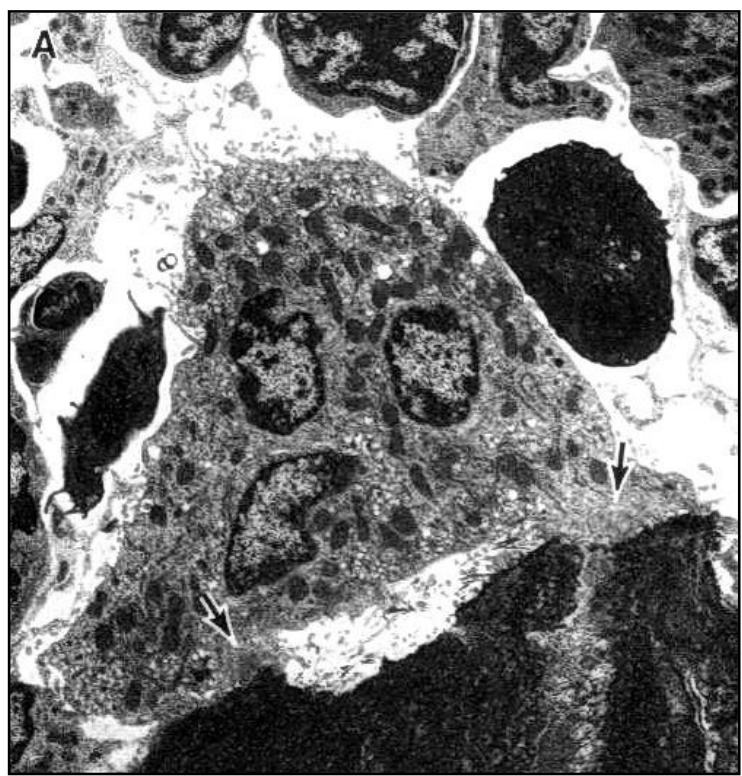

Fig. 7 Electron micrograph of osteoclast of control group at day 5, showing well-developed ruffled border and clear zone. Dense vesicles and vacuoles are scattered in the cytoplasm. X 5000 


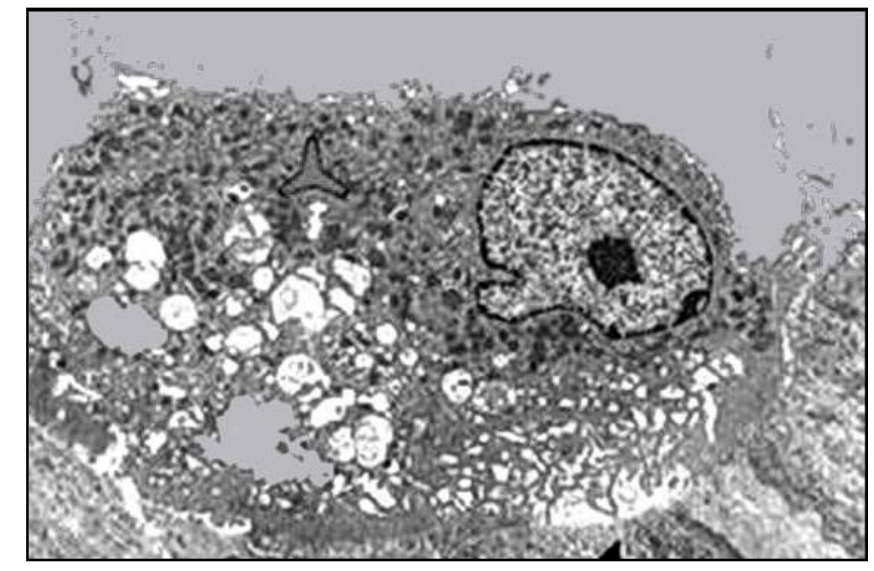

Fig.8 Odontoblasts with characteristic ruffled border, clear zone and vacuolated cytoplasm X6 000

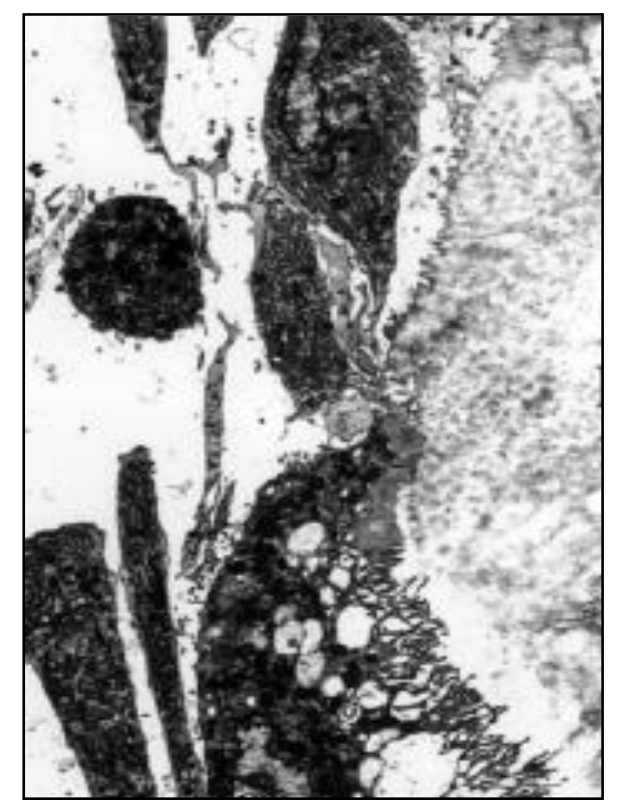

Fig. 9 Electron micrograph of osteoclast at the pressure side at day 5 of bisphosphonate treated rats showing lacks ruffled border $\mathrm{X} 6000$ 


\section{Egyptian}

Orthodontic Journal

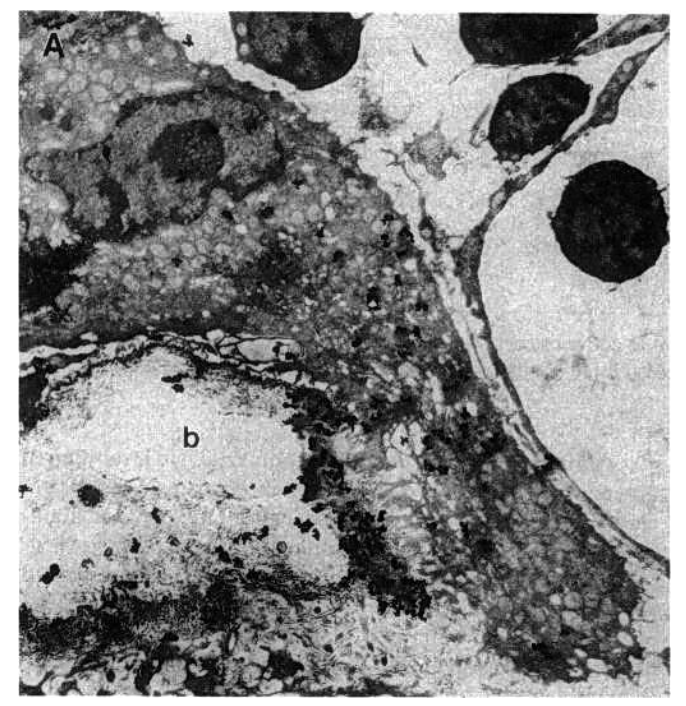

Fig.10 Electron micrograph of osteoclast that lacks both ruffled border and clear zone X 6000

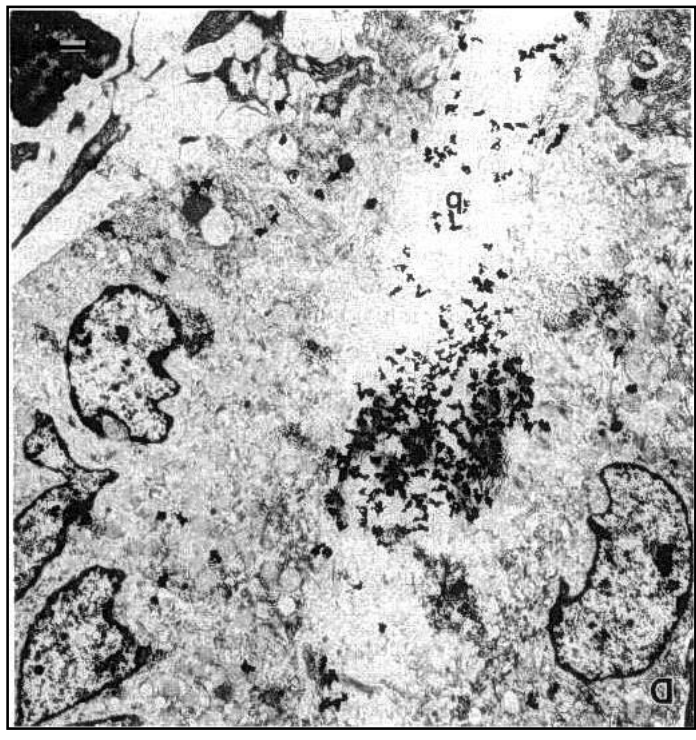

Fig. 11 Degenerated osteoclasts showing absence of the ruffled border, pyknotic nuclei and absence of cytoplasmic vacuoles X 6000 
Egyptian

Orthodontic Journal

\section{DISCUSSION}

One of the shortcomings of orthodontic treatment is the expected relapse after removing the orthodontic appliance and the need for long-term retention. Different procedures are used to ensure stability after orthodontic treatment, such as the fulltime wear of Hawley retainer, bonded lingual retainers, fibrotomy, and many others. Unfortunately, all these measures are subjective and depend on many factors such as the length of retention, the quality of the treatment done, the method of retention and patient cooperation. Clear-cut method for retention is still not available.

Orthodontic tooth movement is mediated by a coupling of bone resorption on the pressure side of the PDL and bone formation on the tension side. ${ }^{(13,14)}$ The role of local bone metabolism associated with orthodontic tooth movement has been considered to be related to the biologic response of periodontal tissues to applied mechanical forces. ${ }^{(14-15)}$

The relapse of orthodontically moved teeth is a physiologic response to force application and has been thought to be caused mainly by contraction of the transseptal fibers. ${ }^{(16,17,18)}$

In the present study, despite only a single systemic dose, there was a significant depression of relapse of the experimentally moved molars. It was more evident after 5 days of elastic band removal. This finding is in accord with the result obtained by several investigators. ${ }^{(4-8,10)}$

The present results showed that the inhibition of the alveolar bone resorption and the corresponding reduction in the relapse of moved teeth is due to the systemic administration of bisphosphonates. Although the mechanism of action is not clear, it might be due to inhibition of bone resorbing activity of osteoclasts. Many osteoclasts on the surface of the alveolar bone in the experimental groups lost their polarity and became round shapes. Some where detached from the surface of the resorption lacunae. A increase in size and number of nuclei, pyknosis and densely stained cytoplasm were also evident in many osteoclasts, in addition, the surface of the resorption lacunae were smoother than those in the control group, indicating decreased bone resorbing activity. Essentially the same histological observations have been reported by previous investigators, ie, loss of ruffled border, ${ }^{(19)}$ increased number of nuclei per cell margins and few lysosomal structures have been reported in osteoclasts in animals treated with bisphosphonates. ${ }^{(20)}$ All these morphologic alternations in osteoclasts support the idea that the prevention of alveolar bone resorption is mediated by the inhibition of osteoclastic function by bisphosphonates. 
Together with the morphologic changes in osteoclasts, the number of osteoclasts tended to decrease with the administration of pamidronate. In the contrary, previous investigators have reported that the number of osteoclasts increased in rats even though bone resorption was blocked. ${ }^{(21)}$ However, other investigators ${ }^{(22-23)}$ have reported that the continuous application of bisphosphonates for a long period often leads to a decrease in the number of osteoclasts, since either the osteoclasts already present are destroyed when they come into contact with or engulf bone containing the bisphosphonate, or the recruitment of new osteoclasts is inhibited.

Although the precise mode of action of bisphosphonate in bone metabolism is not yet clearly understood, pervious studies have suggested that bisphosphonates interact directly with bone cells rather than through some physicochemical process and that they alter the recruitment, function and structure of osteoclasts both in vitro and in vivo. ${ }^{(24)}$

Inhibition of bone resorption by bisphosphonates has been considered to be due a decrease in the number of osteoclasts, at least in cases where resorption depends on the formation of new osteoclasts. Loss of ruffled border, increased number of nuclei per cell, marked vacuolization, more regular cell margins, and fine lysosomal structures have been reported in osteoclasts in animals treated with bisphosphonates. ${ }^{(25)}$

It was found that the bisphosphonate injected rats, most osteoclasts exhibited either irregularly-formed ruffled borders and clear zones or only clear zones of various degrees of extension. Consistent with this result, Sato et al., ${ }^{(26)}$ found that the subcellular localization and expression of both vacuolar-type H+-ATPase and cathepsin $\mathrm{K}$ was significantly decreased in such osteoclasts with impaired ruffled borders and/or only clear zones by bisphosphonate administration

In the drug injected rats, many osteoclasts lacked both prominent ruffled borders and the accumulation of associated vacuoles and were, therefore, assumed to have a decreased bone resorptive capacity. In this regard, it was reported that bisphosphonate induced decrease in lysosomal enzyme release from osteoclasts in more calvarial bones, apparently via a mechanism not related to cytotoxicity. ${ }^{(27)}$ Carano et al., ${ }^{(21)}$ pointed out that bisphosphonate reduced protein synthesis and proton accumulation by cultured osteoclasts. In addition, acid extrusion by osteoclasts was virtually absent where bone had been preincubated with bisphosphonate. ${ }^{(28)}$ These observations strongly suggest selective metabolic and functional impairments of osteoclasts, produced by bisphosphonate, which resulted in reduced bone resorption. 
In this study, the administration of pamidronate through a single injection at a low dosage, as recommended for clinical use, and achieved a diminution in the relapse. Yuki et al., ${ }^{(6)}$ suggested that the systemic subcutaneous administration of bisphosphonate into the subperiosteum of the alveolar bones was found to reduce the extent of relapse of moved molars in rats.

The major effect of bisphosphonate on osteoclasts appears to be depolarization of the osteoclast structure associated with disappearance or irregular formation of ruffled borders and associated vacuoles. Such changes appear to be due to disruption of the cytoskeletal actin rings in BP- treated cultured osteoclasts. ${ }^{(29)}$

In spite of the dose of bisphosphonate (a single injection of $1.5 \mathrm{mg} / \mathrm{kg}$ ) used in this study being similar to that given by Kim et al., ${ }^{(4)}$ and much lower than that used by Hughes et al., (30) (daily injections of $4 \mathrm{mg} / \mathrm{kg}$ for 2 days), the reduction in the relapse movement was apparent. They stated ${ }^{(30)}$ that osteoclast apoptosis is not the only mechanism by which bisphosphonate reduces the bone resorptive activities of osteoclasts.

Further clinical studies are recommended to be done to verify the use of bisphosphonate groups clinically as a biological method to prevent or minimize relapse after orthodontic treatment.

\section{REFERENCES}

1- Pavlin D, Magness M, Zadro R, Goldman ES, Gluhak J. Orthodontically stressed periodontium of transgenic mouse as a model for studying mechanical response in bone: The effect on the number of osteoblasts. Clin Orthod Res 3, 2000:58 55-66

2- Boese LR. Increased stability of orthodontically rotated teeth following gingivectomy in Macaca nemestrina. Am J Orthod 1969;56:273-90.

3- Picton DCA, Moss JP. The part played by the trans-septal fibre system in experimental approximal drift of the cheek teeth of monkey (Macaca Irus). Archs Oral Biol 1973; 18:669-80.

4- Kim TW, Yoshida Y, Yokoya K, Sasaki T. An ultrastructural study of the effects of bisphosphonate administration on osteoclastic bone resorption during relapse of experimentally moved rat molars Am J Orthod Dentofacial Orthop. 1999; 115: 645-53.

5- Montalvetti A, Bailey BN, Martin MB, Severin GW, Oldfield E. Clinical trial of risedronate in Japanese volunteers: a study on the effects of timing of dosing on absorption. J Bone Miner Metab. 2004; 22(2):120-6. 
6- Yuki S, Hideaki S, Yasuhiro K, Yoshinobu S, Takahisa S. Bisphosphonate administration alters subcellular localization of vacuolar-type H+-ATPase and cathepsin $\mathrm{K}$ in osteoclasts during experimental movement of rat molars. Anat Rec. 2000; 260:72-80.

7- Hoon M, Ju-Yong P, Pill-Hoon C. Effects of a bisphosphonate on the expression of bone specific genes after autogenous free bone grafting in rats. $\mathrm{J}$ Periodont Res. 2001; 36: 244.

8- Adachi H, Igarashi K, Mitani H \& Shinoda H. Effects of topical administration of a bisphosphonates (Residronate) on orthodontic movements in rats. J Dent Res 1994; 73: 1478-84.

9- Shoji K, Horiuchi H, Shinoda H. Inhibitory effects of a bisphosphonates (residronate) on experimental periodontits in rats. J Periodont Res 1995; 30: 277-84.

10- Adachi H, Igarashi K, Mitani H, Shinoda H. Anchorage and retentive effects of a bisphosphonate (AHBuBP) on tooth movement in rats. Am J Orthod Dentofacial Orthop 1994; 106: 279-89.

11- Waldo CM, Rothblatt JM. Histologic response to tooth movement in the laboratory rat: procedure and preliminary observation. J Dent Res 1954; 33:481- 6.

12- Lee W. Effect of prostaglandins on tooth movement. Am J Orthod Dentofacial Orthop 1990; 98:231- 41

13- Yokoya K, Sasaki T, Shibasaki Y. Distributional changes of osteoclasts and preosteoclastic cells in periodontal tissues during experimental tooth movement as revealed by quantitative immunohistochemistry of H+ ATPase. J Dent Res 1997; 76: 580 -7.

14- Reitan K. Clinical and histologic observations on tooth movement during and after orthodontic treatment. Angle Orthod 1971; 41: 1-14

15- King GJ, Thiems S. Chemical mediation of bone resorption induced by tooth movement in rat. Arch Oral Biol 1979; 24: 811-815,

16- Davidovitch Z, Shanfeld JL. Cyclic AMP levels in alveolar bone of orthodontically-treated cats. Arch Oral Biol 1975; 20: 567-574

17- Sato M and Grasser W. Effects of bisphosphonates on isolated rate osteoclasts as examined by reflected light microscopy. J Bone Res 1990; 5: 31-40.

18- Brain WE. The effect of surgical transsection of free gingival fibers on the regression of orthodontically rotated teeth in the dog. Am J Orthod 1969;55: 50-70. 
19- Parker GR. Transseptal fibers and relapse following bodily retraction of teeth: a histologic study. Am J Orthod 1972; 61:331-44.

20- Flanagan AM, chambers TJ. Inhibition of bone resorption by bisphosphonates: interactions between bisphosphonates, osteoclasts, and bone. Calcif Tissue Int 1990; 49:407-15.

21- Carano A, teitelbaum SL, konsek JD, Schlesinger PH, Blair HC. Bisphosphonates directly inhibit the bone resorption activity of isolated avian osteoclasts in vitro. J Cline invest 1990; 85:456-61.

22- Alatli I, Hellsing E \& Hammarstrom L. Orthodontically induced root resorption in rat molars after 1-hydroxyethylidene-1, 1-bisphosphonate injection. Acta odontol. Scand 1996; 54: 102-108.

23- Murakami H, Takahashi N, Sasaki T. A possible mechanism of the specific action of bisphosphonates on osteoclasts. Bone 1995; 17; 137-44.

24- Flanagan AM, Chambers TJ. Inhibition of bone resorption by bisphosphonates: interactions between bisphosphonates, osteoclasts, and bone. Calcif Tissue Int 1990; 49:407-15.

25- Sahni M, Guenther HL, Fleisch H, Collin P, Martin TJ. Bisphosphonates act on rat bone resorption through the mediation of osteoblasts. J Clin Invest 1993; 91:2004-11.

26- Sato M, Grasser W, Endo N, Akins R, Simmons H, Thompson DD, et al. Bisphosphonate action: alendronate localization in rat bone and effects on osteoclast ultrastructure. J Clin Invest 1991; 88:2095-105.

27- Sasaki T, Hong MH, Udagawa N, Moriyama Y. Expression of vacuolar HATPase in osteoclasts and its role in resorption. Cell Tissue Res 1994; 278:265-71.

28- Muhlbaucer RC, Bauss F., Schenk R, and Janner M. A potent new bisphosphonate to inhibit bone resorption. J Bone Miner Res 1991; 6: 1003

29- Zimolo Z, Wesolowski G, Rodan GA. Acid extrusion is induced by osteoclast attachment to bone. J Clin Invest 1995; 96: 2277-83.

30- Hughes DE, Wright KR, Uy HL, Sasaki A, Yoneda T, Roodman GD. Bisphosphonates promote apoptosis in murine osteoclasts in vitro and in vivo. J Bone Miner Res 1995; 10:1478-87. 